\title{
Using Adaptive Priority Controls for Service Differentiation in QoS-Enabled Web Servers
}

\author{
Mário Meireles Teixeira ${ }^{2,1}$, Marcos José Santana ${ }^{1}$, and \\ Regina H. Carlucci Santana ${ }^{1 \star}$ \\ 1 University of São Paulo \\ Institute of Mathematics and Computer Science \\ São Carlos, SP, Brazil 13560-000 \\ $\{\mathrm{mjs}, \mathrm{rcs}\} @ i c m c . u s p . b r$ \\ 2 Federal University of Maranhão \\ Department of Informatics \\ São Luís, MA, Brazil 65085-580 \\ mario@icmc.usp.br
}

\begin{abstract}
We propose an architecture for the provision of differentiated services at the web server level. The architecture is validated by means of a simulation model and real web server traces are used as workload. We implement an adaptive algorithm which allows the tuning of the priority level provided and determines how strict the use of priorities will be. The server can then adapt itself to various workloads, an essential feature in a highly dynamic environment such as the Web.
\end{abstract}

\section{Introduction}

The service currently provided on the Internet is based on a best-effort model, which treats all traffic uniformly, without any type of service differentiation or prioritization, a characteristic we find even in the design of critical Internet services, such as the Web. However, not all types of traffic are equivalent or have the same priority to their users [1]. Therefore, it is essential to provide service differentiation with different levels of quality of service (QoS) to different request types [2].

In this paper, we propose a novel architecture for a web server capable of providing differentiated services to its users and applications. We consider two classes of users and analyze the implementation of an adaptive priority mechanism, an innovative solution for service differentiation at the application domain.

There are a few studies in the literature which use priorities for service differentiation [3] 4] 5]. However, all of them are based on some sort of strict priority scheme and do not provide mechanisms for its adaptation, as is the case of our algorithm.

* The authors would like to thank Brazilian funding agencies CAPES, CNPq and FAPESP for their support to the research projects at LaSDPC-ICMC-USP 


\section{Service Differentiating Web Server Model}

In this section, we propose a generic model for a Service Differentiating Web Server (SWDS, in Portuguese) which should be able to provide different levels of service to its clients with quality of service guarantees. Figure 1 describes the proposed architecture, composed of the following modules: a Classifier, an Admission Control module and a cluster of web server processes.

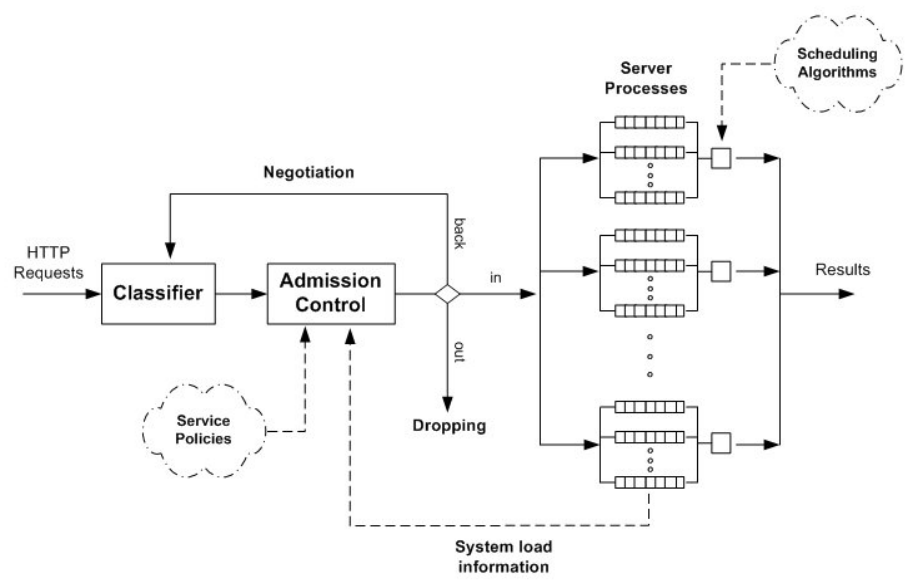

Fig. 1. Service Differentiating Web Server (SWDS)

The Classifier is the element responsible for receiving requests upon arrival at the server and for dividing them into classes following some previously defined criteria. The Admission Control module manages the acceptance of new requests by the server taking into account current service policies and system workload information. In case of system overload, a request may be either rejected (Dropping) or have its QoS requirements downgraded (Negotiation), so that it can be accepted in a lower priority class. After being admitted to the system, the request is assigned to one of the nodes of the web server cluster and is serviced according to the scheduling or service differentiating algorithm currently in operation. After processing, the results are sent back to the clients.

In this work, each cluster node is viewed as a plain web server with a CPU, a disk, a network interface and other resources. The nodes could have also been abstracted as processes, tasks or even CPU's in a parallel computer, since the model does not necessarily imply that the cluster is composed by computers in a distributed system. 


\section{Adaptive Priority Mechanism}

To implement the adaptive algorithm, each server process is defined with a single waiting queue where requests are inserted in strict arrival order. The algorithm uses a look-ahead parameter $(k)$ that specifies the maximum number of positions that will be searched from the head of the queue looking for requests of a given priority (class). If no request of the desired priority is found, the algorithm is repeated for the next lower level and so on. In the worst case, the first request of the queue will be chosen for processing. The higher the value of $k$, the better the treatment given to higher priority requests. For $k=1$, requests will be serviced in strict arrival order, i.e., without any service differentiation.

The model is validated by means of a discrete-event simulation using the SimPack simulation package. We used log files collected from the 1998 World Cup web site [6] for workload generation. We assume four homogeneous web servers in the cluster. Arriving requests are divided into two service classes (high and low priority) with $50 \%$ of the requests in each class. The admission control module is disabled so as not to interfere with the performance evaluation of the algorithm. Therefore, the Classifier works as a dispatcher for the requests and server queues are unlimited.

Initially, we analyzed the behavior of request mean response time for different values of the look-ahead, as shown in Fig. 2. For $k=1$, the curves overlap, since the same treatment is given to both service classes. However, for $k=3000$, the service differentiation becomes evident and the service provided to high priority requests is noticeably better, as initially intended.

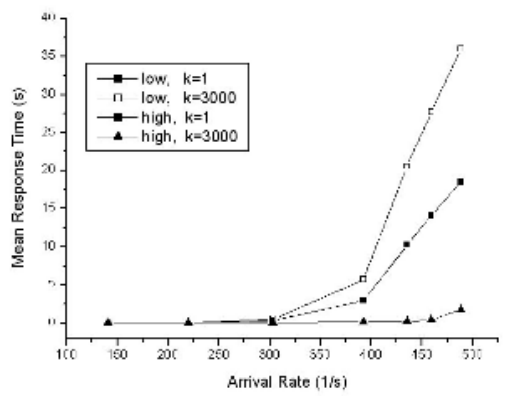

Fig. 2. Request response time using adaptive priority scheduling

The following experiments analyzed the behavior of the ratio of completed high priority requests with respect to the arrival rate. Look-ahead values range from 1 to 4,500. For $k=1$, the service received by both classes of requests is virtually the same. However, higher values of $k$ gradually increase the ratio of high priority requests that reach a successful completion (Fig. 3), to the point where strict priority scheduling is enforced. In this case, the treatment provided to low priority requests becomes much worse. 


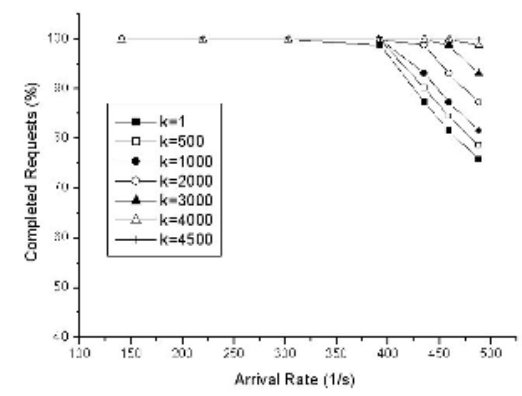

Fig. 3. Ratio of completed requests for different values of the look-ahead

\section{Conclusions}

We proposed an architecture for a service differentiating web server, the SWDS server, which can provide different levels of service to different classes of users. Our model is an evolution from conventional web server architectures, which service clients using an FCFS discipline, without considering the demands of any particular group of users or applications.

We proposed and implemented an adaptive priority mechanism in the SWDS server model, an innovative solution at the application domain. It employs a look-ahead parameter in the cluster's waiting queues in order to fine-tune the prioritization level used by the system. Thus, the server can support varying degrees of QoS-awareness according to the real time system load. The adaptive algorithm brings adaptability to the SWDS server and shifts the workload associated with service differentiation to the cluster nodes, which in turn reduces the workload of the dispatcher and improves system scalability.

\section{References}

1. Dovrolis, C., Ramanathan, P.: A case for relative differentiated services and the proportional differentiation model. IEEE Network (1999)

2. Kant, K., Mohapatra, P.: Scalable Internet servers: Issues and challenges. In: Proceedings of the Workshop on Performance and Architecture of Web Servers (PAWS), ACM SIGMETRICS (2000)

3. Chen, X., Mohapatra, P.: Providing differentiated services from an Internet server. In: Proceedings of the IEEE International Conference on Computer Communications and Networks. (1999) 214-217

4. Eggert, L., Heidemann, J.: Application-level differentiated services for web servers. World Wide Web Journal 3 (1999) 133-42

5. Rao, G., Ramamurthy, B.: DiffServer: Application level differentiated services for web servers. In: Proceedings of the IEEE International Conference on Communications. (2001)

6. Arlitt, M., Jin, T.: Workload characterization of the 1998 World Cup web site. Technical Report HPL-1999-35, HP Laboratories (1999) 